\title{
Pursuing clinician investigator programs in residency
}

\author{
Samir Bidnur, MD \\ Clinician Investigator Program, University of British Columbia, Vancouver, BC, Canada
}

Cite as: Can Urol Assoc J 2016;10(5-6):215. http://dx.doi.org/10.5489/cuaj.3890

A clinician investigator program (CIP) (aka surgeonscientist program [SSP]) is an opportunity offered by most institutions across Canada that allow residents to take time away from surgical residency in order to pursue a formal research program. Their goal is to provide a dedicated research stream in addition to clinical training during a busy surgical residency. This means accomplishing an MSc or a postdoctoral fellowship (if you already have an MSc or $\mathrm{PhD}$ ) within two years of dedicated research time, usually taken after PGY-2 or PGY-3. Additional goals of the CIP are to drive innovation and advancement of knowledge translation in healthcare in Canada and renew clinical academic faculty throughout the country.

I decided to pursue the CIP at the University of British Columbia (UBC) Vancouver Prostate Centre (VPC) because, as residents, with as much as research that is expected and emphasized within our programs, it can be difficult to really experience the depth of research being performed across the street from the hospital by our very own surgeon mentors. VPC is a beehive of research productivity, but almost inaccessible to clinical residents. The CIP is, therefore, the ideal opportunity to be fully immersed in clinical or basic science research for those residents seeking the experience. For me, this meant designing a study, writing the grant to secure funding (and realizing how immensely important this is!), collecting and interpreting data, and finally writing a basic science thesis and manuscript. The skill sets learned include tissue culture techniques, animal modeling techniques, western blotting, polymerase chain reaction, and most importantly, learning how to collaborate effectively with real scientists. The CIP provides the foundation for conducting high-quality research in a busy academic environment. All this helps design future projects, market yourself as a competitive fellow, and ensure that the academic route is right for you.

It is important to stress that residents can create their own experiences in CIP, and CIPs from residents can be quite disparate from each other. It is possible complete a project at your home institution or abroad, be involved with basic sciences or clinical, and work with almost any PI of your choice. The possibilities within the CIP framework are almost endless and, therefore, it is extremely important to select the project, mentor, and setting where each applicant can be most successful. This is the most important step prior to initiating any project.

To be a successful researcher in medicine, you need two things: Time and mentorship. This is what I've heard throughout residency from many successful surgeon scientists. For the urology resident interested in pursuing an academic path, CIPs provide this research time and mentorship during residency, establishing strong foundations on which to build upon during fellowship and an academic career. Although they add two extra years of residency training, this investment can pay dividends throughout a research career. I thank the UBC CIP program for delivering such a strong platform to promote research in residency.

Competing interests: The author declares no competing financial or personal interests.

Correspondence: Dr. Samir Bidnur, Clinician Investigator Program, University of British Columbia, Vancouver, BC, Canada; bidnursa@gmail.com 